\title{
Glomerular Regeneration: When Can the Kidney Regenerate from Injury and What Turns Failure into Success?
}

\author{
Anna Peired ${ }^{a} \quad$ Elena Lazzeria Laura Lasagnia Paola Romagnania, b \\ ${ }^{a}$ Excellence Centre for Research, Transfer and High Education for the Development of De Novo Therapies \\ (DENOTHE), University of Florence, and b Pediatric Nephrology Unit, Meyer Children's Hospital, Florence, Italy
}

\section{Key Words}

Renal progenitors · Podocytes · Kidney regeneration

\begin{abstract}
Background: For many years, the glomerulus was considered incapable of regeneration. However, experimental and clinical evidence challenged this concept and showed that glomerular injury and even glomerulosclerosis can undergo regression under certain circumstances. The problem with glomerular regeneration is centered around the podocyte, a highly specialized cell that is the critical constituent of the glomerular filtration barrier. Summary: Podocytes are characterized by a complex cytoskeleton that makes them unable to proliferate. Thus, once their depletion reaches a specific threshold, it is considered to be irreversible. The discovery of cells with the aptitude to differentiate into podocytes in the adult kidney, i.e. renal progenitor cells (RPCs), was a critical step in understanding the mechanisms of glomerular repair. Accumulating evidence suggests that a tight regulation of many different signaling pathways, such as Notch, Wnt, and microRNA, is involved in a correct regenerative process and that an altered regulation of these same signaling pathways in RPCs triggers the generation of focal segmental glomerulosclerosis lesions. In particular, regeneration is severely impaired by proteinuria, when albumin sequesters
\end{abstract}

retinoic acid and blocks RPC differentiation in podocytes. Key Messages: RPC maintenance and differentiating potential are regulated by complex mechanisms that can be implemented following glomerular injury and can be manipulated to activate regeneration for therapeutic purposes. A better understanding of the phenomenon of glomerular regeneration paves the way for the prevention and treatment of glomerular diseases.

(c) 2014 S. Karger AG, Basel

\section{Introduction}

Although primary injury to each of the somatic cell types in the glomerular tuft is associated with some form of glomerular disease, injuries to endothelial and mesangial cells are repaired via the proliferation of adjacent cells. By contrast, podocytes are highly specialized, terminally differentiated cells arrested in the G0 phase of the cell cycle, with a limited capacity for cell division. They can be forced to reorganize their actin cytoskeletal fibers to try to enter cell division. However, changes in the complex cytoskeletal architecture of the foot processes lead to their effacement, cell detachment, or death by mitotic catastrophe, with disruption of the glomerular filtration barrier (GFB) and ensuing proteinuria [1]. A depletion of

\section{KARGER}

E-Mail karger@karger.com

www.karger.com/nee
(C) 2014 S. Karger AG, Basel

$1660-2129 / 14 / 1262-0070 \$ 39.50 / 0$ 
up to $20 \%$ of the podocytes can be compensated for, while a loss of $20-40 \%$ of podocytes results in scarring and a loss of more than $60 \%$ leads to glomerulosclerosis [2]. Recent results have conclusively demonstrated that when the podocyte depletion is below $20 \%$, generation of novel podocytes can occur after injury, as shown in lineagetracing studies in conditional transgenic mice [3]. By contrast, podocytes can undergo hypertrophy, that is an increase in cell size, in an attempt to cover the underlying glomerular basement membrane in exposed areas where neighboring cells have detached or died, a phenomenon which is not associated with de novo podocyte generation [3].

However, when the injury exceeds a certain threshold, podocyte hypertrophy reveals itself to be an unfit strategy over time, as the loss of podocytes and segmental sclerosis lead to a decreased ultrafiltration capacity and single nephron glomerular filtration rate [1]. Nevertheless, remission of the disease and regression of glomerulosclerosis have been observed in experimental animals and in patients with diabetic and nondiabetic nephropathies, suggesting that regeneration can occur to some extent [4]. The first evidence of glomerular regeneration was presented in a study by Fioretto et al. [5] published in 1998, which showed regression of glomerular lesions in diabetic patients after 10 years and paved the way for a fastgrowing line of research on glomerular regeneration.

\section{How to Counter Podocyte Loss}

Since lost podocytes cannot be replaced by proliferating podocytes, other mechanisms of compensation must be implemented in the glomerulus. The first cells proposed as being able to replace podocytes were bone marrow-derived cells due to their capacity to differentiate into a wide range of cell types. Initial studies suggested that bone marrow-derived cells contribute to renal epithelial regeneration following kidney injury [6]. However, more recent studies have shown the incapacity of bone marrow-derived cells to differentiate into epithelial cells and argue that their beneficial effects in kidney diseases are limited to the secretion of regenerative and prosurvival paracrine factors $[7,8]$.

The existence of renal progenitor cells (RPCs) and their capacity to replace injured podocytes were first reported in an adult human kidney in our laboratory $[9,10]$. These cells were identified by the expression of CD24, a marker of renal embryonic cells, and CD133, a marker of several types of adult stem cells. Both markers were coex- pressed by a subset of parietal epithelial cells (PECs) localized at the urinary pole of Bowman's capsule in adult kidneys and characterized by self-renewal, a high clonogenic efficiency, and multidifferentiation potential. RPCs were further described to form a heterogeneous and hierarchical population of undifferentiated cells including pluripotent cells and an intermediate population of transitional cells - or podocyte progenitors - expressing both podocyte and progenitor markers and capable of differentiating into podocytes [10]. When injected into severe combined immunodeficiency mouse models of focal segmental glomerulosclerosis (FSGS), human RPCs could engraft, replace lost podocytes, and improve proteinuria, suggesting that RPCs can differentiate into podocytes and promote podocyte regeneration. In addition, using genetic tagging of PECs in an inducible transgenic mouse model, Appel et al. [11] demonstrated that PECs can differentiate into podocytes during kidney growth.

Recently, Pippin et al. [12] showed that renin lineage cells of the juxtaglomerular apparatus have regeneration capacity and were able to replace both PECs and podocytes on the glomerular tuft in a murine model of FSGS, suggesting that they may represent upstream mesenchymal progenitors. Renin lineage cells are located outside the glomerulus in the preglomerular vascular wall, which could be an advantage for escaping intraglomerular damage, but it is presently unclear how they would cross the parietal and/or glomerular basement membrane [13].

\section{When Regeneration Works: Successful Renal Progenitor Cell Differentiation Allows Podocyte Repopulation}

Tissue homeostasis and wound repair require epithelial stem cells to execute different programs of terminal differentiation. To reach that goal, stem cells must be directed toward a particular cell lineage by their microenvironment. The canonical Notch signaling pathway is an evolutionarily conserved mechanism that regulates decisions to self-renew and/or differentiate through closerange, cell-cell interactions in a variety of different tissues [14], including the kidney. In vitro experiments have shown that Notch activation in human RPCs leads to entry into the S-phase of the cell cycle and successive cell division, while its downregulation is essential for RPC differentiation into podocytes [15]. Accordingly, inhibition of Notch signaling throughout the regenerative phases led to aggravation of the albuminuria and glomerulosclerosis in a murine model of Adriamycin nephropa- 
thy [15]. Furthermore, prolonged Notch blocking in a genetically engineered mouse model of FSGS reduced RPC proliferation and the formation of RPC hyperplastic lesions but worsened podocyte loss and proteinuria [16]. Therefore, a tight regulation of the Notch pathway compensates for a disrupted GFB with progressive podocyte loss. Taken together, the evidence shows that Notch is a key regulator not only of kidney development but also of glomerular repair.

Likewise, data suggest that the Wnt pathway plays a role in podocyte maintenance. The Wnt pathway is a highly conserved signaling pathway that regulates cellular interactions at various stages of embryonic and fetal development in several organs, including cell fate decisions, branching morphogenesis, coordinated proliferation, cell polarization, and basement membrane synthesis. Conditional $\beta$-catenin knockout mice, in which a Wnt downtarget has been deleted, form abnormal kidneys and have reduced renal function. A great percentage of glomeruli have small, underdeveloped capillary tufts and well-differentiated podocytes that replace PECs in Bowman's capsule. Capillaries toward the outer side of these podocytes attempt to resemble glomerular capillaries. Tracing nephrogenesis in embryonic conditional $\beta$-catenin knockout mice revealed that the Wnt pathway drives the differentiation of RPCs into podocytes in the parietal layer of the S-shaped body by direct lineage switch. Taken together, these findings demonstrate that $\beta$-catenin/Wnt signaling is important during the late stages of nephrogenesis and for the lineage specification of RPCs into podocytes [17].

Among other factors implicated in the control of RPC differentiation into podocytes are retinoids. Indeed, it was recently shown by Zhang et al. [18] that retinoic acid is able to drive the expression of podocyte markers in RPCs and ultimately lead to an increase in podocyte number in an experimental model of FSGS in mice.

Finally, in the last few years, a large number of studies have presented evidence that microRNAs, a class of small noncoding RNAs, play a key role in stem cell self-renewal and differentiation. Recent data identified miR-1915 and miR-1225-5p as regulators of the stemness and repair capacity of RPCs through modulation of CD133, Pax2, and other important markers of RPCs [19]. Interestingly, members of the miR-30 family were shown to protect podocytes from injury by inhibiting Notch1 and p53 [20], while miR-193a was identified as being responsible for WT1 downregulation in podocytes and the resulting cell death in FSGS [21], illustrating the wide involvement of microRNAs in glomerular physiology.

\section{When Regeneration Fails: Impaired Renal Progenitor Cell Differentiation into Podocytes Drives FSGS}

When the podocyte loss is severe, regeneration is not observed, while hyperplastic cellular lesion and matrix production do occur, with consequent glomerular scarring. RPCs are also involved in this process and they represent the major constituent of FSGS lesions. Interestingly, there is now evidence that the same mechanisms that drive RPC differentiation into podocytes are also involved in the generation of FSGS lesions when they are not appropriately regulated. In particular, impaired silencing of the Notch pathway after RPC differentiation into podocytes caused the podocytes to override the G2/M checkpoint, resulting in cytoskeleton disruption and cell death by mitotic catastrophe. While Notch signaling was almost completely absent in healthy adult kidneys, it was markedly increased in the glomerular epithelial cells of patients affected by glomerular diseases. Inhibition of Notch signaling in a mouse model of FSGS resulted in an improvement of albuminuria and a reduced podocyte loss in the initial phases of chronic glomerular injury. This study demonstrates that Notch-regulated balance between podocyte loss and RPC-driven regeneration influences the outcome of glomerular disorders [15]. Inducible expression of the intracellular domain of Notch in podocytes was sufficient to cause albuminuria, glomerulosclerosis, and death of the animal through the activation of $\mathrm{p} 53$. On the other hand, podocyte-specific deletion of the Notch transcriptional partner RBPJ or pharmacological inhibition of Notch cleavage protected mice from proteinuria and podocyte loss in rodent models of diabetic nephropathy and FSGS [22]. Therefore, aberrant Notch signaling appears to be behind the phenotypic changes of RPCs in the pathogenesis of FSGS.

Wnt ligands were upregulated in podocytes of experimental models of podocyte injury, as well as in human biopsy samples of patients affected by glomerular diseases, including acute kidney injury, glomerular diseases, diabetic nephropathy, fibrosis, and cystic kidney diseases. Several studies have used genetic approaches to show the effects of activation of Wnt signaling in podocytes by deleting or constitutively expressing the downstream transcriptional regulator $\beta$-catenin only in podocytes. While all of the mice developed normally and were healthy, indicating that the $\mathrm{Wnt} / \beta$-catenin pathway is dispensable in development and homeostasis, the studies revealed that $\beta$-catenin activity was harmful in models of acute glomerular injury [23]. 
Fig. 1. Scheme showing the blocking effect of albumin on RARE activation induced by retinoids in RPCs. In the presence of mild proteinuria, retinoids promote RPC differentiation in podocytes through RARE activation. In the presence of high proteinuria, albuminuria sequesters RA and impairs podocyte regeneration, promoting the generation of FSGS lesions.

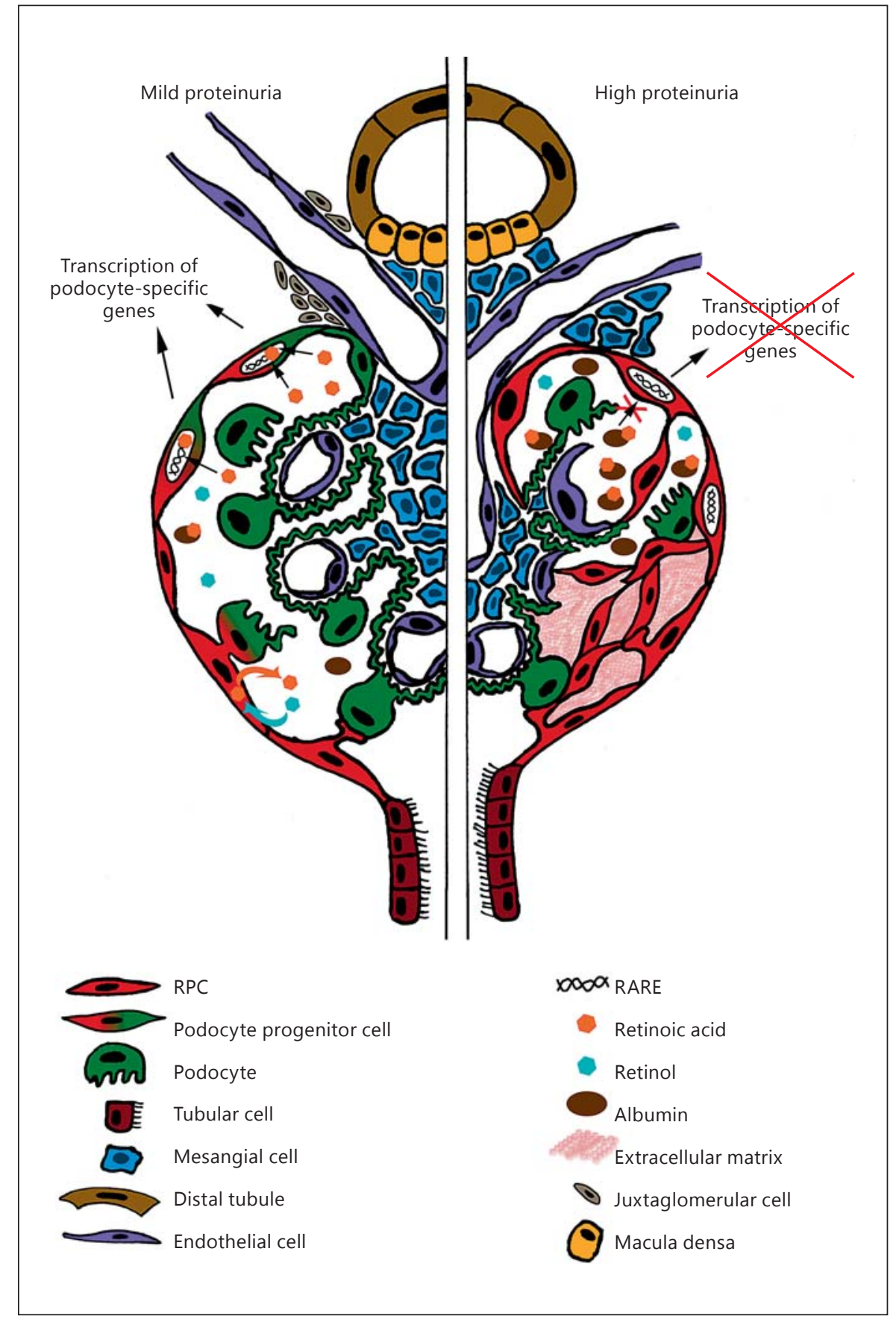

The correlation between proteinuria and progressive renal damage, characterized by podocyte loss, is well established in diabetic and nondiabetic nephropathies. Sustained antiproteinuric treatments with ACE inhibitors slow down disease progression and can even induce regression of glomerulosclerosis, associated with an increase in podocyte number, both in experimental and clinical settings $[3,24]$. While depleted podocytes can be replaced by RPCs, the actual regeneration observed during glomerular diseases is limited. Recently we proposed that proteinuria impairs RPC differentiation in podocytes and is responsible for the lack of glomerular regeneration (fig. 1) [25]. In primary cultures of RPCs, exposure to transferrin and IgG induced apoptotic cell death, whereas exposure 
to human serum albumin inhibited their differentiation into podocytes by sequestering RA and preventing the retinoic acid response element (RARE)-mediated transcription of podocyte-specific genes. To verify in vivo that albumin overload impaired the RA-induced podocyte differentiation of RPCs, we used a transgenic mouse model of Adriamycin nephropathy in which the Cre-loxP system allowed genetic tagging of podocytes and therefore permitted direct quantification of the podocyte loss. In severe combined immunodeficiency mice affected with Adriamycin nephropathy, blocking endogenous RA synthesis with retinaldehyde dehydrogenase (RALDH) inhibitors increased proteinuria, glomerular injury, and animal death. In RARE-LacZ transgenic animals, albuminuria reduced the RA bioavailability and impaired RARE activation in RPCs and the acquisition of podocyte markers by renal progenitors, even if the RALDH activity was not modified. Treatment with RA reduced the proteinuria and increased podocyte numbers, as assessed in serial kidney biopsies, by reversing the RARE-lacZ activity in RPCs. This study suggests that, during glomerular disorders, albumin lost through the damaged GFB represents not only a predictive factor for progression but also a cause for glomerulosclerosis. Consistently, recent results have confirmed that podocyte regeneration occurs when the podocyte loss is below $20 \%$ and the proteinuria is transient [3]. Taken together, these results explain why reducing proteinuria delays the progression of $\mathrm{CKD}$, and they provide the biological rationale for the clinical use of antiproteinuric drugs to induce regression of glomerular disorders.

\section{Conclusions}

Podocyte loss is a key event in progression toward glomerulosclerosis. Mechanisms of podocyte depletion have been described, exploring in particular the fact that podocytes are quiescent cells that cannot reorganize their actin cytoskeleton to reenter the cell cycle and replicate without undergoing detachment or mitotic catastrophe. Nevertheless, glomerular regeneration occurs, as has been observed in various experimental and clinical settings, and can be potentially driven by RPC-mediated podocyte repopulation. Accumulating evidence suggests that a tight regulation of many different signaling pathways, such as Notch, Wnt, and microRNA, is involved in a correct regenerative process. However, when these same mechanisms do not work properly, glomerular diseases progress toward glomerulosclerosis and an altered regulation of these same pathways in RPCs triggers the generation of FSGS lesions. In particular, regeneration is severely impaired by proteinuria, when albumin sequesters retinoic acid and blocks RPC differentiation in podocytes. This phenomenon is not ineluctable, as underlined by the occurrence of glomerular regeneration when protein-lowering drugs such as ACE inhibitors are used, and can be even pharmacologically enhanced, turning failure into success.

\section{References}

1 Lasagni L, Lazzeri E, Shankland SJ, Anders HJ, Romagnani P: Podocyte mitosis - a catastrophe. Curr Mol Med 2013;13:13-23.

2 Wiggins RC: The spectrum of podocytopathies: a unifying view of glomerular diseases. Kidney Int 2007;71:1205-1214.

3 Wanner N, Hartleben B, Herbach N, Goedel M, Stickel N, Zeiser R, Walz G, Moeller M, Grahammer F, Huber TB: Unraveling the role of podocyte turnover in glomerular aging and injury. J Am Soc Nephrol 2014, E-pub ahead of print.

-4 Remuzzi G, Benigni A, Remuzzi A: Mechanisms of progression and regression of renal lesions of chronic nephropathies and diabetes. J Clin Invest 2006;116:288-296.

5 Fioretto P, Steffes MW, Sutherland DE, Goetz FC, Mauer M: Reversal of lesions of diabetic nephropathy after pancreas transplantation. N Engl J Med 1998;339:69-75.
6 Poulsom R, Forbes SJ, Hodivala-Dilke K, Ryan E, Wyles S, Navaratnarasah S, Jeffery R, Hunt T, Alison M, Cook T, Pusey C, Wright NA: Bone marrow contributes to renal parenchymal turnover and regeneration. J Pathol 2001;195:229-235.

-7 Sagrinati C, Ronconi E, Lazzeri E, Lasagni L, Romagnani P: Stem-cell approaches for kidney repair: choosing the right cells. Trends Mol Med 2008;14:277-285.

-8 Zoja C, Garcia PB, Rota C, Conti S, Gagliardini E, Corna D, Zanchi C, Bigini P, Benigni A, Remuzzi G, Morigi M: Mesenchymal stem cell therapy promotes renal repair by limiting glomerular podocyte and progenitor cell dysfunction in Adriamycin-induced nephropathy. Am J Physiol Renal Physiol 2012; 303:F1370-F1381.

-9 Sagrinati C, Netti GS, Mazzinghi B, Lazzeri E, Liotta F, Frosali F, Ronconi E, Meini C, Gacci
M, Squecco R, Carini M, Gesualdo L, Francini F, Maggi E, Annunziato F, Lasagni L, Serio M, Romagnani S, Romagnani P: Isolation and characterization of multipotent progenitor cells from the Bowman's capsule of adult human kidneys. J Am Soc Nephrol 2006;17: 2443-2456.

10 Ronconi E, Sagrinati C, Angelotti ML, Lazzeri E, Mazzinghi B, Ballerini L, Parente E, Becherucci F, Gacci M, Carini M, Maggi E, Serio M, Vannelli GB, Lasagni L, Romagnani S, Romagnani P: Regeneration of glomerular podocytes by human renal progenitors. J Am Soc Nephrol 2009;20:322332.

11 Appel D, Kershaw DB, Smeets B, Yuan G, Fuss A, Frye B, Elger M, Kriz W, Floege J, Moeller MJ: Recruitment of podocytes from glomerular parietal epithelial cells. J Am Soc Nephrol 2009;20:333-343. 
12 Pippin JW, Sparks MA, Glenn ST, Buitrago S, Coffman TM, Duffield JS, Gross KW, Shankland SJ: Cells of renin lineage are progenitors of podocytes and parietal epithelial cells in experimental glomerular disease. Am J Pathol 2013;183:542-557.

13 Grahammer F, Wanner N, Huber T: Podocyte regeneration: who can become a podocyte? Am J Pathol 2013;183:333-335.

14 Koch U, Lehal R, Radtke F: Stem cells living with a Notch. Development 2013;140:689704.

15 Lasagni L, Ballerini L, Angelotti ML, Parente E, Sagrinati C, Mazzinghi B, Peired A, Ronconi E, Becherucci F, Bani D, Gacci M, Carini M, Lazzeri E, Romagnani P: Notch activation differentially regulates renal progenitors proliferation and differentiation toward the podocyte lineage in glomerular disorders. Stem Cells 2010;28:1674-1685.

16 Ueno T, Kobayashi N, Nakayama M, Takashima Y, Ohse T, Pastan I, Pippin JW, Shankland SJ, Uesugi N, Matsusaka T, Nagata M: Aberrant Notch1-dependent effects on glomerular parietal epithelial cells promotes collapsing focal segmental glomerulosclerosis with progressive podocyte loss. Kidney Int 2013;83: 1065-1075.
17 Grouls S, Iglesias DM, Wentzensen N, Moeller MJ, Bouchard M, Kemler R, Goodyer P, Niggli F, Gröne HJ, Kriz W, Koesters R: Lineage specification of parietal epithelial cells requires $\beta$-catenin/Wnt signaling. J Am Soc Nephrol 2012;23:63-72.

18 Zhang J, Pippin JW, Vaughan MR, Krofft RD, Taniguchi Y, Romagnani P, Nelson PJ, Liu ZH, Shankland SJ: Retinoids augment the expression of podocyte proteins by glomerular parietal epithelial cells in experimental glomerular disease. Nephron Exp Nephrol 2012;121:e23-e37.

19 Sallustio F, Serino G, Costantino V, Curci C, Cox SN, De Palma G, Schena FP: miR-1915 and miR-1225-5p regulate the expression of CD133, PAX2 and TLR2 in adult renal progenitor cells. PLoS One 2013;8:e68296.

$20 \mathrm{Wu}$ J, Zheng C, Fan Y, Zeng C, Chen Z, Qin W, Zhang C, Zhang W, Wang X, Zhu X, Zhang M, Zen K, Liu Z: Downregulation of microRNA-30 facilitates podocyte injury and is prevented by glucocorticoids. J Am Soc Nephrol 2014;25:92-104.
21 Gebeshuber CA, Kornauth C, Dong L, Sierig R, Seibler J, Reiss M, Tauber S, Bilban M, Wang S, Kain R, Böhmig GA, Moeller MJ, Gröne HJ, Englert C, Martinez J, Kerjaschki D: Focal segmental glomerulosclerosis is induced by microRNA-193a and its downregulation of WT1. Nat Med 2013;19:481-487.

22 Niranjan T, Bielesz B, Gruenwald A, Ponda MP, Kopp JB, Thomas DB, Susztak K: The Notch pathway in podocytes plays a role in the development of glomerular disease. Nat Med 2008;14:290-298.

23 Kato H, Gruenwald A, Suh JH, Miner JH, Barisoni-Thomas L, Taketo MM, Faul C, Millar SE, Holzman LB, Susztak K: Wnt/ $\beta$ catenin pathway in podocytes integrates cell adhesion, differentiation, and survival. J Biol Chem 2011;286:26003-26015.

24 Rizzo P, Perico N, Gagliardini E, Novelli R, Alison MR, Remuzzi G, Benigni A: Nature and mediators of parietal epithelial cell activation in glomerulonephritides of human and rat. Am J Pathol 2013;183:1769-1778.

- 25 Peired A, Angelotti ML, Ronconi E, la Marca G, Mazzinghi B, Sisti A, Lombardi D, Giocaliere E, Della Bona M, Villanelli F, Parente E, Ballerini L, Sagrinati C, Wanner N, Hubber TB, Liapis H, Lazzeri E, Lasagni L, Romagnani P: Proteinuria impairs podocyte regeneration by sequestering retinoic acid. J Am Soc Nephrol 2013;24:1756-1768. 